\title{
ÁCIDO FÓLICO, PREVENÇÃO DE DEFEITOS DO TUBO NEURAL E FATORES ASSOCIADOS: UMA REFLEXÃO
}

\author{
ÁCIDO FÓLICO, PREVENCIÓN DE DEFECTOS DEL TUBO NEURAL Y FACTORES \\ ASOCIADOS: UNA REFLEXIÓN
}

\author{
FOLIC ACID, PREVENTION OF NEURAL TUBE DEFECTS AND ASSOCIATED \\ FACTORS: A REFLECTION
}

\author{
Alessandra Bernadete TROVÓ DE MARQUI ${ }^{1}$
}

\begin{abstract}
RESUMO: Tem como objetivo discutir os fatores associados ao uso de ácido fólico durante a gestação. Estudo descritivo-reflexivo com embasamento teórico elaborado a partir de artigos científicos atualizados. Apesar dos benefícios do ácido fólico e mesmo se tratando de uma recomendação mundial, a prevalência de consumo deste suplemento é, ainda, insatisfatória. Alguns estudos têm mostrado que mulheres com gravidez não planejada, menor escolaridade e nível socioeconômico, sem companheiro e com pré-natal insuficiente são mais susceptíveis a não consumir esse suplemento durante a gestação. A suplementação com ácido fólico é uma intervenção imprescindível, atuando na prevenção primária dos defeitos do tubo neural. Assim, políticas públicas que venham a incentivar ainda mais a suplementação materna com ácido fólico são de grande valia, uma vez que reduzem a morbimortalidade neonatal. Também é necessário o planejamento de ações que visem minimizar a ação dos fatores associados ao uso de ácido fólico durante a gestação.
\end{abstract}

PALAVRAS-CHAVE: Ácido fólico. Defeitos do tubo neural. Gravidez. Enfermagem obstétrica. Educação em saúde.

RESUMEN: Tiene como objetivo discutir los factores asociados al uso de ácido fólico durante la gestación. Estudio descriptivo-reflexivo con base teórica elaborado a partir de artículos científicos actualizados. Apesar de los beneficios del ácido fólico e incluso si se trata de una recomendación mundial, la prevalencia de consumo de este suplemento es todavía insatisfactoria. Algunos estudios han demostrado que las mujeres con embarazo no planificado, menor escolaridad y nivel socioeconómico, sin compañero y con prenatal insuficiente, son más susceptibles a no consumir ese suplemento durante la gestación. La suplementación con ácido fólico es una intervención imprescindible, actuando en la prevención primaria de los defectos del tubo neural. Así, las políticas públicas que fomenten aún más la suplementación materna con ácido fólico son de gran valor, ya que reducen la morbimortalidad neonatal. También es necesario la planificación de acciones que busquen minimizar la acción de los factores asociados al uso de ácido fólico durante la gestación.

PALABRAS CLAVE: Ácido fólico. Defectos del tubo neural. Embarazo. Enfermería obstétrica. Educación en salud.

${ }^{1}$ Universidade Federal do Triângulo Mineiro (UFTM), Uberaba - MG - Brasil. Professora associada da disciplina de genética. Departamento de patologia, genética e evolução. ORCID: http://orcid.org/0000-0003-2361-5174. Email: alessandratrovo@hotmail.com 
ABSTRACT: Aims to discuss factors associated with the use of folic acid during gestation. A descriptive-reflective study with a theoretical basis elaborated from updated scientific articles. Despite the benefits of folic acid and, even if it is a worldwide recommendation, the prevalence of this supplement consumption is still unsatisfactory. Some studies have shown that women with unplanned pregnancies, lower schooling and socioeconomic status, without partners and with insufficient prenatal care are less likely to consume this supplement during gestation. Folic acid supplementation is an essential intervention, acting in the primary prevention of neural tube defects. Thus, public policies that further encourage maternal supplementation with folic acid are of great value, since they reduce neonatal morbidity and mortality. It is also necessary to plan actions that aim to minimize the action of the factors associated with the use of folic acid during gestation.

KEYWORDS: Folic acid. Neural tube defects. Pregnancy. Obstetric nursing. Health education.

\section{Introdução}

O ácido fólico, também conhecido como vitamina B9 ou folato, é predominantemente encontrado nas folhas/vegetais verde escuras (couve, brócolis, aspargo, espinafre, rúcula, mostarda), feijão, lentilhas, grão de bico, frutas cítricas, fígado e vísceras animais. Esses alimentos constituem fontes naturais dessa vitamina e o seu preparo deve ser criterioso, pois parte significativa do ácido fólico é oxidada (entre 50\% e 95\%) e destruída quando o alimento passa pelo processo de cozimento ou preparo. É uma vitamina solúvel na água, daí a perda durante o processamento dos alimentos. Esse composto tem função fundamental no processo de multiplicação celular sendo, portanto, indispensável durante a gravidez.

É consenso na literatura científica o papel efetivo dessa vitamina na prevenção de malformações congênitas, tais como os defeitos do tubo neural (DTN). Os DTN são anomalias congênitas resultantes do fechamento incompleto ou incorreto do tubo neural e surgem entre a terceira e quarta semana do desenvolvimento embrionário. Eles incluem a anencefalia, espinha bífida e encefalocele, sendo a primeira incompatível com a vida e as outras associadas com alta morbidade e mortalidade perinatal. A etiologia dos DTN é associada a uma herança multifatorial decorrente da interação entre fatores genéticos e ambientais. Os DTN podem ser identificados no nascimento por meio do preenchimento do campo 34, "malformação congênita e/ou anomalia cromossômica", na Declaração de Nascidos Vivos (DNV) do Sistema de Informações sobre Nascidos Vivos (SINASC). É importante o preenchimento correto da DNV para se estabelecer a real prevalência das anomalias congênitas em nosso país. 
No Brasil, políticas públicas de suplementação de ácido fólico foram implementadas visando prevenir os DTN e englobam a fortificação desse micronutriente nas farinhas de trigo e milho (BRASIL, 2002), bem como a suplementação diária desse composto para gestantes no período periconcepcional, conforme Manual Técnico de Pré-natal e Puerpério. Resultados positivos em relação à primeira iniciativa foram obtidos em um estudo conduzido no Estado de São Paulo com uso do SINASC, que analisou a prevalência e distribuição espacial de DTN, antes e após a fortificação das farinhas de trigo e milho com ácido fólico. A prevalência diminuiu $35 \%$, de 0,57 para 0,37 por mil nascidos vivos após a fortificação (OR = 0,65 ; IC95\%: 0,59-0,72) (FUJIMORI et al., 2013).

No entanto, essas ações preventivas de saúde podem ter sua efetividade comprometida pela diversidade de hábitos alimentares regionais e gestações não planejadas. A gravidez não planejada constitui um fator de risco para malformações congênitas, segundo dados do Estudo Colaborativo Latino-Americano de Malformações Congênitas (ECLAMC). Um estudo brasileiro em crianças e adolescentes com espinha bífida mostrou que 78\% das mães declararam que sua gravidez não foi planejada e 80\% delas afirmaram não ter feito uso de ácido fólico nos primeiros meses gestacionais (GAÍVA; CORRÊA; SANTO, 2011). Brasileiras grávidas jovens (<20 anos) exibiram elevada prevalência de recém-nascidos com DTN, explicada pela não suplementação pré-concepcional de ácido fólico em gravidezes não planejadas, como é característico nas adolescentes (REIS et al., 2015).

Ainda em relação à prevenção dos DTN, um estudo na cidade de Juiz de Fora, Minas Gerais, com 118 profissionais de saúde (95 médicos e 23 enfermeiros atuantes em obstetrícia), avaliou o nível de conhecimento sobre a utilização de ácido fólico para a prevenção dos DTN (CONCEIÇÃO et al., 2012). Não houve diferença entre o nível de conhecimento quando comparado à formação dos mesmos e entre as duas classes de profissionais avaliados. A maioria $(94,1 \%)$ dos profissionais disseram conhecer o papel do ácido fólico, contudo, 64,2\% relataram não saber quando iniciar a suplementação no pré-natal (CONCEIÇÃO et al., 2012). Esse achado é preocupante, uma vez que tais profissionais atuam no período gravídico-puerperal. Já em relação às gestantes pesquisadas, $96,2 \%$ relataram ter ouvido falar de folato. Destas, apenas $27,6 \%$ conheciam o importante papel do folato na prevenção de DTN e somente $20,1 \%$ delas havia ingerido ácido fólico durante o período periconcepcional (NOSRAT; SEDEHI; GOLALIPOUR, 2012). Essa última pesquisa revelou que os serviços de saúde $(54,5 \%)$ foram a principal fonte de informação sobre os benefícios do folato, reforçando a necessidade de conhecimento adequado desses profissionais para promoção da saúde materno-infantil (NOSRAT; SEDEHI; GOLALIPOUR, 2012). 
Outras anomalias congênitas nas quais o ácido fólico tem efeito protetor incluem a prevenção da ocorrência de fissuras labiopalatinas não sindrômicas (SILVA et al., 2019). Os DTN e as fissuras labiopalatinas ocorrem em períodos embriologicamente semelhantes e, além disso, o desenvolvimento das estruturas faciais se dá a partir de células oriundas da crista neural, originárias do fechamento do tubo neural (SILVA et al., 2019).

Os DTN representam uma importante causa de morbidade e mortalidade infantil. Cerca de $50 \%$ das crianças afetadas morrem no primeiro ano de vida e as que sobrevivem apresentam incapacidade física e/ou intelectual importante que requer reabilitação prolongada e de alto custo para a família e a sociedade. Estudo recente (FRANÇA et al., 2017) que investigou as principais causas de morte na infância no Brasil mostrou que as anomalias congênitas, que ocupavam a $5^{a}$ posição em 1990 , passaram a figurar como $2^{a}$ principal causa em 2015 , embora tenha ocorrido discreta diminuição das taxas nos anos analisados — de 3,31 para 3,06/1.000 nascidos vivos (FRANÇA et al., 2017).

Apesar dos benefícios do ácido fólico, mesmo se tratando de uma recomendação mundial, a prevalência de consumo deste suplemento é ainda insatisfatória. Estudos brasileiros têm mostrado que mulheres com gravidez não planejada, menor escolaridade e nível socioeconômico, sem companheiro e com pré-natal insuficiente são mais susceptíveis a não consumir esse suplemento durante a gestação (BARBOSA et al., 2011; ESPOLADOR et al., 2015; AMARAL et al., 2016; LINHARES et al., 2017). Conhecer os fatores que influenciam o consumo do ácido fólico durante a gestação permite que sejam traçadas estratégias para minimizar os efeitos da não adesão à essa prática, extremamente importante para proteção do Sistema Nervoso Central do feto.

Diante do exposto, o objetivo do presente estudo é discutir os fatores associados ao uso de suplemento de ácido fólico durante a gestação, uma vez que está bem consolidado na literatura os benefícios desse composto para a prevenção dos DTN. Para embasamento teórico dessa reflexão, foram utilizados, preferencialmente, artigos científicos atualizados que abordavam essa temática.

\section{Fatores associados ao uso de suplemento de ácido fólico durante a gestação}

Os resultados apresentados no Quadro 1 mostram que os fatores que elevaram o uso do folato foram: número de consultas do pré-natal superior a sete; o fato da gestação ter sido planejada; maior nível de escolaridade e idade entre as gestantes; idade gestacional menor que 11-12 semanas e; conhecimento sobre o ácido fólico (Barbosa et al., 2011; Espolador et al., 
2015; Amaral et al., 2016; Linhares et al., 2017). Um desses estudos mencionou a existência de negligência por parte dos profissionais de saúde que acompanham as gestantes, tanto no sentido de prescreverem, quanto de monitorarem o consumo desse composto Barbosa et al. (2011). Outra pesquisa conclui que é necessária a implantação de campanhas mais eficazes, direcionadas, sobretudo, para as mulheres com menor nível socioeconômico, Linhares et al. (2017).

Quadro 1 - Sumário dos principais estudos brasileiros que avaliaram os fatores associados à suplementação com ácido fólico na gestação.

\begin{tabular}{|c|c|c|c|}
\hline Referência & Objetivo & Casuística & Principais resultados \\
\hline Barbosa et al. (2011) & $\begin{array}{l}\text { Estimar a } \\
\text { prevalência de } \\
\text { consumo de } \\
\text { suplemento de } \\
\text { ácido fólico } \\
\text { durante a gestação } \\
\text { e identificar os } \\
\text { fatores associados } \\
\text { ao seu consumo. }\end{array}$ & $\begin{array}{l}280 \text { mulheres da } \\
\text { cidade de } \\
\text { Diamantina, MG. }\end{array}$ & $\begin{array}{l}\text { Mulheres com menor escolaridade, } \\
\text { adolescentes e com número de consultas de } \\
\text { pré-natal (inferior a sete) apresentaram, } \\
\text { respectivamente, } 1,61 \text { (IC95\%=1,34-1,93), } \\
1,18 \text { (IC } 95 \%=1,03-1,35) \text { e } 1,18 \\
\text { (IC95\%=1,02-1,37) mais chances de não } \\
\text { consumir o suplemento durante a gestação. A } \\
\text { prevalência de consumo de ácido fólico entre } \\
\text { as gestantes foi baixa e associada à idade, } \\
\text { escolaridade materna e ao número de } \\
\text { consultas pré-natal. }\end{array}$ \\
\hline Espolador et al. (2015) & $\begin{array}{l}\text { Identificar os } \\
\text { fatores associados } \\
\text { ao uso da } \\
\text { suplementação do } \\
\text { ácido fólico na } \\
\text { gestação. }\end{array}$ & $\begin{array}{l}120 \text { gestantes do pré- } \\
\text { natal da cidade de } \\
\text { São José do Rio } \\
\text { Preto, SP. }\end{array}$ & $\begin{array}{l}\text { Concluiu-se que ter mais idade, possuir } \\
\text { prescrição da suplementação do ácido fólico e } \\
\text { a idade gestacional menor que } 12 \text { semanas } \\
\text { foram fatores que influenciaram a ingestão do } \\
\text { ácido fólico. }\end{array}$ \\
\hline Amaral et al. (2016) & $\begin{array}{l}\text { Avaliar a } \\
\text { prevalência do uso } \\
\text { de suplementação } \\
\text { com ácido fólico } \\
\text { em gestantes e } \\
\text { identificar fatores } \\
\text { associados à não } \\
\text { realização dessa } \\
\text { prática. }\end{array}$ & $\begin{array}{l}316 \text { puérperas da } \\
\text { cidade de Joinville, } \\
\text { SC. }\end{array}$ & $\begin{array}{l}\text { A conclusão do ensino superior aumentou em } \\
3,5 \text { vezes a probabilidade de suplementação } \\
\text { com folato [OR } 3,5 \text { (IC } 95 \%, 1,1-11,1)] \text {. O } \\
\text { planejamento da gravidez e o início do pré- } \\
\text { natal anterior à } 11^{\text {a }} \text { semana também } \\
\text { aumentaram as chances de consumo de ácido } \\
\text { fólico em [OR } 2,0 \text { (IC } 95 \%, 1,2-3,5)] \text { e [OR } \\
2,2 \text { (IC } 95 \%, 1,2-4,3)], \text { respectivamente. O } \\
\text { conhecimento sobre ácido fólico foi o maior } \\
\text { preditor de suplementação, aumentando-a em } \\
10 \text { vezes [OR } 10,1 \text { (IC } 95 \%, 5,0-20,9)] . \\
\text { Dentre as mulheres que usaram folato, } \\
\text { somente } 22(19,6 \% \text { iniciaram antes da } \\
\text { gravidez. Além disso, o tempo médio de uso } \\
(3,3 \text { meses } \pm 2,7) \text { esteve abaixo do } \\
\text { recomendado. As chances de adesão à } \\
\text { suplementação com folato foram maiores em } \\
\text { gestantes que apresentaram maior } \\
\text { escolaridade, planejamento da gravidez, } \\
\text { início do pré-natal anterior à } 11^{\text {a }} \text { semana e } \\
\text { conhecimento sobre o papel do ácido fólico. }\end{array}$ \\
\hline Linhares et al. (2017) & $\begin{array}{l}\text { Identificar a } \\
\text { prevalência e os } \\
\text { fatores associados } \\
\text { ao uso do } \\
\text { suplemento de } \\
\text { ácido fólico na } \\
\text { gestação. }\end{array}$ & $\begin{array}{l}2.685 \text { puérperas da } \\
\text { cidade de Rio } \\
\text { Grande, RS. }\end{array}$ & $\begin{array}{l}\text { A prevalência de uso de ácido fólico foi de } \\
54,2 \% \text {. Os fatores associados ao uso de ácido } \\
\text { fólico foram: cor da pele branca, viver com } \\
\text { companheiro, maior escolaridade e renda } \\
\text { familiar, ser primípara, ter planejado a gravi- } \\
\text { dez, ter seis ou mais consultas de pré-natal e } \\
\text { ter iniciado o pré-natal no primeiro trimestre } \\
\text { de gestação. }\end{array}$ \\
\hline
\end{tabular}

* MG: Minas Gerais, SC: Santa Catarina, SP: São Paulo, RS: Rio Grande do Sul

Fonte: elaborado pelo autor. 
O planejamento da gravidez é um fator de difícil interferência, pois, o tubo neural, estrutura precursora do cérebro e da medula espinhal, fecha-se entre $22^{\circ}$ e $28^{\circ}$ dias após a concepção, período no qual muitas mulheres desconhecem ainda seu estado gravídico. $\mathrm{O}$ fechamento deste tubo é essencial para a formação da calota craniana e da coluna vertebral. As mulheres que tomam o ácido fólico depois do resultado do teste de gravidez correm o risco desta anomalia já estar em desenvolvimento, pois a época de aparecimento deste tipo de malformação é muito precoce. A época do estabelecimento dos diversos tipos de malformações fetais é: defeitos do tubo neural - 28 dias; defeitos do septo ventricular cardíaco - 42 dias; lábio leporino - 36 dias; fenda palatina - 47 a 72 dias. Nas gestações planejadas, o contato entre a paciente e o médico é geralmente mais extenso, o que favorece a orientação acerca dos complexos vitamínicos protetores do recém-nascido (AMARAL et al., 2016).

Outro achado interessante mostrou empoderamento a partir do conhecimento científico possuído pelas gestantes, algo que pode ser considerado condição necessária para o uso profilático do folato (AMARAL et al., 2016). No entanto, ainda é necessário realizar campanhas de conscientização para mulheres em idade fértil no que se refere à promoção do uso de ácido fólico profilático. Tais atividades devem ser estendidas aos profissionais de saúde envolvidos na assistência pré-natal e à sociedade em geral, através de cursos de capacitação e divulgação na mídia.

A escolaridade é outro fator importante a ser discutido. Gestantes com maior escolaridade possuem maior acesso à informações e, consequentemente, maior conhecimento do papel protetor do folato na prevenção dos DTNs. Já a baixa escolaridade geralmente está relacionada ao início mais tardio de pré-natal e menor frequência de consultas (BARBOSA et al., 2011). Nas gestantes com menor escolaridade, é imprescindível que os profissionais de saúde utilizem uma linguagem de fácil entendimento, ou seja, popularizem o conhecimento científico.

Os resultados apresentados no Quadro 1 geram alguns questionamentos: a finalidade do diagnóstico pré-natal está sendo atingida? Ou seja, as orientações sobre o uso da suplementação vitamínico-mineral estão sendo abordadas adequadamente, visando proporcionar uma gestação tranquila, saudável e segura? 


\section{Ácido fólico e Enfermagem}

O profissional de enfermagem, especificamente aquele que atua no ciclo gravídicopuerperal, tem papel inquestionável na promoção da saúde materno-infantil. Somado a isso, devemos destacar que, a prevenção das anomalias congênitas se faz, sobretudo, na saúde pública preventiva, ou seja, no nível primário. Nessa perspectiva, a Enfermagem poderá atuar em Educação em Saúde por meio da promoção de campanhas educativas sobre a importância do ácido fólico na prevenção dos DTN, com foco em mulheres em idade fértil. No entanto, estudo prévio mostrou importante nível de desconhecimento de médicos e enfermeiros obstetras sobre a utilização do ácido fólico, relativos ao tempo de utilização, o início da suplementação e a dosagem ideal, pontos relevantes na prevenção dos DTN. Assim, a capacitação desses profissionais é necessária para minimizar os riscos de tais anomalias (CONCEIÇÃO et al., 2012).

Ainda, considerando os elevados custos financeiros relacionados ao tratamento e acompanhamento dos pacientes com DTN, além das graves consequências pessoais, familiares e sociais, contrapondo-se à eficiência e ao baixo custo da prevenção, esperamos que este trabalho possa, de forma efetiva, contribuir para a elaboração de outras estratégias de saúde pública, que interfiram diretamente na prevenção dessas graves anomalias congênitas (CONCEIÇÃO et al., 2012).

\section{Considerações finais}

A suplementação com ácido fólico é uma intervenção imprescindível, atuando na prevenção primária dos defeitos do tubo neural. Assim, políticas públicas que venham a incentivar ainda mais a suplementação materna com ácido fólico são de grande valia, uma vez que reduzem a morbimortalidade neonatal. Também é necessário o planejamento de ações que visem minimizar a ação dos fatores associados ao uso de ácido fólico durante a gestação.

\section{REFERÊNCIAS}

AMARAL, A. R. DO et al. Fatores associados à suplementação com ácido fólico em gestantes da cidade de Joinville, SC. Arquivos Catarinenses de Medicina, v. 45, n. 3, p. 7183, 2016.

BARBOSA, L. et al. Factors associated with folic acid use during pregnancy. Revista Brasileira de Ginecologia e Obstetrícia, v. 33, n. 9, p. 246-251, 2011. 
BRASIL. Ministério da Saúde. Agência Nacional de Vigilância Sanitária. Resolução RDC n. 344, de 13 de dezembro de 2002. Regulamento técnico para fortificação das farinhas de trigo e milho com ferro e ácido fólico. Diário Oficial da União, 2002. p. 18.

CONCEIÇÃO, R. C. et al. Knowledge of physicians and obstetric nurses about the prevention of neural tube defects. Ciência \& Saúde Coletiva, v. 17, n. 10, p. 2795-2803, 2012.

ESPOLADOR, G. M. et al. Identificação dos fatores associados ao uso da suplementação do ácido fólico na gestação. Revista de Enfermagem do Centro Oeste Mineiro, v. 5, n. 2, p. 1552-1561, 2015.

FRANÇA, E. B. et al. Leading causes of child mortality in Brazil, in 1990 and 2015: estimates from the Global Burden of Disease study. Revista Brasileira de Epidemiologia, v. 20, Suppl 01, p. 46-60, 2017.

FUJIMORI, E. et al. Prevalence and spatial distribution of neural tube defects in São Paulo State, Brazil, before and after folic acid flour fortification. Cadernos de Saúde Pública, v. 29, n. 1, p. 145-154, 2013.

GAÍVA, M. A. M.; CORRÊA, E. R.; SANTO, E. A. R. DO E. Perfil clínico-epidemiológico de crianças e adolescentes que vivem e convivem com espinha bífida. Revista Brasileira de Crescimento e Desenvolvimento Humano, v. 21, n. 1, p. 99-110, 2011.

LINHARES, A. O.; CESAR, J. A. Suplementação com ácido fólico entre gestantes no extremo Sul do Brasil: prevalência e fatores associados. Ciência \& Saúde Coletiva, v. 22, n. 2, p. 535-542, 2017.

NOSRAT, S. B.; SEDEHI, M.; GOLALIPOUR, M. J. Knowledge and practice of urban Iranian pregnant women towards folic acid intake for neural tube defect prevention. Journal of Pakistan Medical Association, v. 62, n. 8, p. 785-789, 2012.

REIS, L. V. DOS. et al. Anomalias congênitas identificadas ao nascimento em recémnascidos de mulheres adolescentes. Acta Médica Portuguesa, v. 28, n. 6, p. 708-714, 2015.

SILVA, C. M. et al. O papel do ácido fólico na prevenção das fissuras labiopalatinas não sindrômicas: uma revisão integrativa. Brazilian Applied Science Review, v. 3, n. 1, p. 641658, 2019.

\section{Como referenciar este artigo}

TROVÓ DE MARQUI, Alessandra Bernadete. Ácido fólico, prevenção de defeitos do tubo neural e fatores associados: uma reflexão. Temas em Educ. e Saúde, Araraquara, v. 15, n. 2, p. 186-193, jul./dez. 2019. e-ISSN 2526-3471. DOI: https://doi.org/10.26673/tes.v15i2.12712 